\title{
Knowledge, Attitude and Practice towards Menstrual Hygiene among Adolescent Girls: A case study from Dehradun, Uttarakhand
}

\author{
Himani Nautiyal*, Arshita Kumari, Kriti Ranjana, Sanjay Singh \\ Department of Pharmacology, Siddhartha Institute of Pharmacy, Dehradun, 248001, Uttarakhand, India. \\ *Corresponding Author Email: himanibpharma2011@gmail.com
}

Received: 11.10.2021; Revised: 18.12.2021; Accepted:19.12.2021

OSociety for Himalayan Action Research and Development

\begin{abstract}
Menstrual hygiene is a prime key of prevention from various reproductive tract infections, which may lead to severe health consequences among girls sometimes infertility, cervical and endometrial cancer.The study aims to find the knowledge, attitude and practice regarding menstrual hygiene among adolescent girls in a Government school of Dehradun.The study was conducted on 200 girls who willingly participated in the survey, from the girl's school in Dehradun. Girls were interviewed with the help of structured questionnaire and data was evaluated using SPSS version 21.0 licensed software. One-way ANOVA test was used for assessing the data P value $(<0.05)$ was considered significant. The majority of girls $143(71.5 \%)$, were $>16$ years of ages, belonging to middle class $68(34 \%)$ and joint family $136(66 \%)$ set up. In spite of average knowledge $55.83 \%$ about menstruation, $145(72.5 \%)$ girls were aware about menstruation process, Girls agreed they can enter kitchen (41/91\%), can take bath (54.54\%), and they agreed they can sleep on same bed $50.50 \%$ during menstruation, need not be separated at this specific time indicates positive attitude and $66.33 \%$ girls have overall good practice during menstruation. .
\end{abstract}

Key Words- Menstrual hygiene, Adolescent girls, Knowledge, Practice, Government Girls School, Dehradun

\section{Introduction}

Menstruation is a natural unique process, which is an unavoidable and important stage of women's life, where her body undergoes different reproductive changes (Deshpande et al 2018). Menstruation process involved shedding of uterus lining under strict hormonal control secreted by pituitary gland (Poureslami, Osati-Ashtiani 2002, ). The onset of menstruation is known as Menarche, the onset age is different for every girl from 13-17 years based on studies (Chumlea et al 2003, Aryeetey et al 2011, Dambhare et al 2012, Tunau et al 2012, Gultie et al 2014, Yasmin et al 2013). Due to lack for scientific knowledge in different cultures and religions, menstruating females perceive psychological and social burden in terms of shame, disgust and fear ${ }^{9}$. Lack of 
Himalayan J. Soc. Sci. \& Humanities ISSN: 0975-9891

Vol. 16, (2021) 35-48

DOI: https://doi.org/10.51220/hjssh.v16i1.3

menstrual knowledge may lead to low hygiene and malpractice during menstruation. This unhygienic behavior is responsible for various reproductive tract and urinary tract infections along with some pelvic inflammatory conditions also (Thomas et al 2001, Barathalakshmi et al 2014).

Menstrual hygiene term indicates menstrual healthcare needs and requirement during menstrual cycle (Sommer, Sahin 2013). According to United Nation "Menstrual hygiene management in females is defined as usage of clean absorbent material for the collection and absorption of menstrual blood, which can be privately changed during menstruation period, availability of soap and water for washing the body whenever required and the disposal facilities for the used absorbent material (UNESCO 2013, Reid, Bruce 2003). Every year approximately 10\% females affect with reproductive tract infection, urinary tract infection and bacterial vaginosis, almost $75 \%$ females have history of such infection in their reproductive ages. The risk factors involved in these infections are pregnancy and menstrual unhygienic (Goel et.al. 2018).

Girls with sound knowledge about menstruation during their menarche can effectively manage menstruation, which is a milestone for a better reproductive health later in their life. Different misconceptions related to menstrual practice are available in India, females and girls have restrictions for certain activities during this period ${ }^{15}$. Mothers and Elder Sisters are first to interact with girls regarding menstruation, so it is very important to evaluate their knowledge, attitude related to this process and practical approach regarding this specific period.

\section{Material and Method}

This school based study was conducted by interviewing students of (class $9^{\text {th }}-12^{\text {th }}$ ) of Government Girls Schools around Rajpur Road in Dehradun during November-December, 2019 by student pharmacists. All girl students were informed about this study and those who agreed to enroll themselves were interviewed after getting signed informed consent form. A pre-designed questionnaire was used for this purpose; it was prepared in Hindi and English language for better understanding of students. The questionnaire included socio-economic status and educational qualification of parents, 6 knowledge related questions, 8 attitude related questions and 6 practice related question during menstruation. Participants were assured about confidentiality of data. After interview participants were educated about facts and importance of hygiene during menstruation by student pharmacist. UNICEF guidelines were applied for the practice 
Himalayan J. Soc. Sci. \& Humanities ISSN: 0975-9891

Vol. 16, (2021) 35-48

DOI: https://doi.org/10.51220/hjssh.v16i1.3

assessment(Reid , Bruce 2003). The completely filled questionnaire data was coded for minimal errors. One way ANOVA test was used for assessing the data using SPSS version 21.0 licensed software. $\mathrm{P}$ value $(<0.05)$ was considered significant.

\section{Result}

Socio-demographic Profile: The socio-demographic profile of the respondents is shown in (Table 1). In this study, the majority, $143(71.5 \%)$, were $>16$ years of ages, (mean age of study population $=15.38 \pm 1.08$ years $)$, were Hindu, $176(88 \%)$, and were living in a joint family $136(66 \%)$. In this study most of the students were studying in class $12^{\text {th }}, 58(29 \%)$. Most of their parents $68(34 \%)$ belonging to the middle social class i.e. 3196-6390 rupees/ month salary. The education status of parents was primary education for both mother and father i.e 79 (39.5\%) and 108(54\%) respectively. Mostly 93 (46.5\%) father belongs to private job and mothers were involved as home maker $94(47 \%)$.

Awareness toward menstruation: Awareness towards menstruation among school girls is presented in Table 2. According to this study 145(72.5\%) girls were aware about menstruation process, out of 200 participants 148 (74.0\%) girls have heard about menstruation before attaining menarche. Most of the girls were not aware about

Table 1: Subject distribution as per socio-demographic profiles

\begin{tabular}{|c|c|}
\hline Variable $\quad(200)$ & Number (\%) \\
\hline $\begin{array}{lc}\text { I. } & \text { Age } \\
\text { a. } & \text { more than } 16 \\
\text { b. } & \text { Less than } 16\end{array}$ & $\begin{array}{l}143(71.5) \\
57(28.5)\end{array}$ \\
\hline $\begin{array}{llll} & & \text { II. } & \text { Class } \\
\text { a. } & 9 \text { th } & & \\
\text { b. } & 10 \text { th } & & \\
\text { c. } & 11 \text { th } & & \\
\text { d. } & 12 \text { th } & & \\
\text { ln }\end{array}$ & $\begin{array}{l}54(27) \\
50(25) \\
38(19) \\
58(29)\end{array}$ \\
\hline \begin{tabular}{ll} 
& \multicolumn{1}{c}{ III. Economic status } \\
a. & $(>=6391$ \\
b. & $3196-6390$ \\
c. & $1917-3195$ \\
d. & $(959-1916$ \\
e. & $(=<958$
\end{tabular} & $\begin{array}{l}62((31) \\
68(34) \\
30(15) \\
10(7.5) \\
30(15)\end{array}$ \\
\hline $\begin{array}{l}\text { IV. Type of family } \\
\text { a. Nuclear } \\
\text { b. Joint }\end{array}$ & $\begin{array}{l}64(32) \\
136(66)\end{array}$ \\
\hline
\end{tabular}

OSHARAD 
Himalayan J. Soc. Sci. \& Humanities ISSN: 0975-9891

Vol. 16, (2021) 35-48

DOI: https://doi.org/10.51220/hjssh.v16i1.3

\begin{tabular}{|c|c|}
\hline $\begin{array}{ll} & \text { V. Religion } \\
\text { a. } & \text { Hindu } \\
\text { b. } & \text { Muslim } \\
\text { c. } & \text { Other }\end{array}$ & $\begin{array}{l}176(88) \\
18(09) \\
06(03)\end{array}$ \\
\hline \begin{tabular}{ll} 
& \multicolumn{1}{c}{ VI. Father's Education status } \\
a. Secondary or higher \\
b. Middle \\
c. Primary \\
d. Illiterate
\end{tabular} & $\begin{array}{l}50(25) \\
23(11.5) \\
108(54) \\
19(9.5)\end{array}$ \\
\hline \begin{tabular}{ll} 
& \multicolumn{1}{c}{ VII. Mother's Education status } \\
a. & Secondary or higher \\
b. & Middle \\
c. & Primary \\
d. & Illiterate
\end{tabular} & $\begin{array}{l}21(10.5) \\
53(26.5) \\
79(39.5) \\
47(23.5)\end{array}$ \\
\hline $\begin{array}{ll} & \text { VIII. Occupation of father } \\
\text { a. } & \text { Government job } \\
\text { b. } & \text { Private Job } \\
\text { c. } & \text { Self employed }\end{array}$ & $\begin{array}{l}23(11.5) \\
93(46.5) \\
84(42)\end{array}$ \\
\hline \begin{tabular}{ll} 
& \multicolumn{1}{c}{ IX. Occupation of Mother } \\
a. & Government job \\
b. & Private Job \\
c. & Self employed \\
d. & Homemaker \\
\end{tabular} & $\begin{array}{l}16(08) \\
37(18.5) \\
53(26.5) \\
94(47)\end{array}$ \\
\hline $\begin{array}{cl}\text { X. } & \text { Primary source of information } \\
\text { a. } & \text { Mother } \\
\text { b. } & \text { Sister } \\
\text { c. } & \text { Relative } \\
\text { d. } & \text { Neighbor } \\
\text { e. } & \text { Teacher } \\
\end{array}$ & $\begin{array}{l}121(61.1 \%) \\
10(5.05 \%) \\
10(5.05 \%) \\
06(03 \%) \\
53(26.76 \%)\end{array}$ \\
\hline $\begin{array}{l}\text { XI. Reaction to first menstruation } \\
\text { a. Scared } \\
\text { b. Usual } \\
\text { c. Discomfort }\end{array}$ & $\begin{array}{l}48(24.24 \%) \\
96(48.48 \%) \\
56(28 \%)\end{array}$ \\
\hline
\end{tabular}

term "Menstrual Hygiene" as only 49 (24.5\%) answered positive for this question. Again only 47

$(23.5 \%)$ students were aware about unhygienic condition of menstrual blood.

Table 2: Table demonstrating distribution of awareness regarding menstruation

\begin{tabular}{|l|l|l|}
\hline Question & Yes (n \%) & No (n \%) \\
\hline Awareness regarding menstruation process & $145(72.5 \%)$ & $53(26.5 \%)$ \\
\hline Heard about menstruation before attaining menarche & $148(74.0 \%)$ & $52(26.0 \%)$ \\
\hline Knew about menstrual hygiene & $49(24.5 \%)$ & $151(75.5 \%)$ \\
\hline Knew that there is foul smelling during menstruation & $49(24.5 \%)$ & $151(75.5 \%)$ \\
\hline Knew that menstrual blood is unhygienic & $47(23.5 \%)$ & $153(76.5 \%)$ \\
\hline
\end{tabular}


Himalayan J. Soc. Sci. \& Humanities ISSN: 0975-9891

Vol. 16, (2021) 35-48

DOI: https://doi.org/10.51220/hjssh.v16i1.3

Knowledge about Menstrual Hygiene: Table 3 describes knowledge towards menstruation among school girls; the overall knowledge was $55.83 \%$ as per this study. Only 50 girls (25\%) out of 200 girls have knowledge about correct source of menstrual bleeding. 147 girls (73.5\%) have accurate knowledge that this is a physiological process. As per this study, 20 girls out of 200 $(10 \%)$ said, it is a Curse of god, 22 girls out of $200(11 \%)$ says menstrual bleeding is resulted due to some Disease and only 11 girls out of $200(5.5 \%)$ said menstruation results because of some sin done by a female. The $150(75 \%)$ participants in this study answered correctly regarding Normal interval between menstrual cycles i.e. (28-35 days), only 50 (25\%) participants answered incorrectly i.e. (40-45days). The 53 (26.5\%) girls answered positive regarding pregnancy during menstruation, while $147(73.5 \%)$ responded negative for this question. The 141 (70.5\%) girls have correct knowledge that poor menstrual hygiene can leads to infections, 129 (64.5\%) girls were known to ovulation period i.e. a time when females are more fertile during menstrual cycle.

Attitude regarding menstruation : Table 4 illustrate attitude of girls regarding menstruation. As per this study only $14(7.07 \%)$ girls were strongly disagree about entering the temple during periods. $83(41.91 \%)$ girls agrees a woman can enter kitchen or cook food during menstruation. Also 108 (54.54\%) girls agrees for taking bath during menses, whereas 121 (61.1\%) girls i.e. more than $50 \%$ percent of study population agree for hair wash during menstruation. 100 (50.50\%) girls agree for using same bed with others for sleep during menstruation. A menstruating woman can touch pickle was agreed by $103(52.02 \%)$ girls, In this study 84 $(41.41 \%)$ girls agreed that a woman need not avoid any foods during menstruation, when asked about sexual intercourse during menstruation, 97 (48.98\%) girls have disagreed in this study.

Table 3: Knowledge of girl students regarding Menstruation

\begin{tabular}{|l|l|l|l|}
\hline Variable & Number (\%) & $\begin{array}{l}\text { Correct } \\
\text { knowledge score }\end{array}$ & $\begin{array}{c}\% \\
\text { Correct score }\end{array}$ \\
\hline Source of menstrual bleeding & $50(25)$ & & \\
Uterus & $81(40.5)$ & 50 & $25 \%$ \\
Urinary bladder & $79(39.5)$ & & \\
Don't know & & & \\
\hline
\end{tabular}


Himalayan J. Soc. Sci. \& Humanities ISSN: 0975-9891

Vol. 16, (2021) 35-48

DOI: https://doi.org/10.51220/hjssh.v16i1.3

\begin{tabular}{|c|c|c|c|}
\hline $\begin{array}{l}\text { What is the menstruation process } \\
\text { Physiological } \\
\text { Curse of god } \\
\text { Disease } \\
\text { Result of some sin }\end{array}$ & $\begin{array}{l}147(73.5) \\
20(10) \\
22(11) \\
11(5.5)\end{array}$ & 147 & $73.5 \%$ \\
\hline $\begin{array}{l}\text { Normal interval between menstrual cycles } \\
\text { Correct( } 28-35 \text { days) } \\
\text { (40-45days) }\end{array}$ & $\begin{array}{l}150(75) \\
50(25)\end{array}$ & 150 & $75 \%$ \\
\hline $\begin{array}{l}\text { Can woman ever be pregnant during menstrual } \\
\text { flow? } \\
\text { Yes } \\
\text { No }\end{array}$ & $\begin{array}{l}53(26.5) \\
147(73.5)\end{array}$ & 53 & $26.5 \%$ \\
\hline $\begin{array}{l}\text { Poor menstrual hygiene can lead to infections } \\
\text { Yes } \\
\text { No }\end{array}$ & $\begin{array}{l}141(70.5) \\
59(29.5)\end{array}$ & 141 & $70.5 \%$ \\
\hline $\begin{array}{l}\text { Is there a period women are most fertile? } \\
\text { Yes } \\
\text { No }\end{array}$ & $\begin{array}{l}129(64.5) \\
71(35.5)\end{array}$ & 129 & $64.5 \%$ \\
\hline $\begin{array}{l}\text { Percentage } \\
55.83 \%\end{array}$ & & & knowledge \\
\hline
\end{tabular}

\section{Practice toward Menstruation:}

Menstruation practice of school girls was depicted in Table 5. The overall good practice percentage was $66.33 \%$ in this study. Only 159 girls (79.5\%) out of 200 girls were using sanitary napkins for soakage of menstrual bleeding, only 41 girls $(20.5 \%)$ were using clothes for the same. The Storage of soakage material was also practiced well by 133 girls (66.5\%) out of 200 participants as the unused cloths and pads were wrapped in tissue or plastic bag for further use. Only 41 girls (21\%) stored sanitary pads in the storage area, whereas 26 girls (13\%) were ill practising the storage of pads with other household material.

The changing frequency of soakage material was practiced well by $103(51.5 \%)$ girls as these girls were changing it for every 3-4 hrs. Also 51 (26\%) girl's changing frequency was at every 6 hrs. $46(23 \%)$ girls were worse in practicing it as they were changing it at 12 hrs. 125 girls $(62.5 \%)$ were practicing good for soakage material disposal as they were wraping a pad in a paper to make a clean package in the bin so it can be burned later. Whereas 75 girls (37.5\%) were involved in wrong practice as the pads were disposed in an open dustbin. $131(65.5 \%)$ girls were practicing Bath during Menstruation, whereas only 09 girls (4.5\%) were not bathing during 
Himalayan J. Soc. Sci. \& Humanities ISSN: 0975-9891

Vol. 16, (2021) 35-48

DOI: https://doi.org/10.51220/hjssh.v16i1.3

menstruation and 60 girls (30\%) were occasionally bathing during menstruation. Washing of genitals after micturition was practised by $145(72.5 \%)$ girls, 35 (17.5\%) girls were not washing it during menstruation which can lead to different infections, and $20(10 \%)$ girls were doing it occasionally.

Table 4: Table demonstrating distribution of attitude regarding menstruation

\begin{tabular}{|l|l|l|l|l|l|}
\hline Question & $\begin{array}{l}\text { Strongly } \\
\text { disagree (0) }\end{array}$ & Disagree (1) & Neutral (2) & Agree (3) & $\begin{array}{l}\text { Strongly agree } \\
(4)\end{array}$ \\
\hline $\begin{array}{l}\text { A woman can enter } \\
\text { temple/pray during } \\
\text { menstruation }\end{array}$ & $14(7.07 \%)$ & $72(36.36 \%)$ & $20(10.0 \%)$ & $\begin{array}{l}65 \\
(32.82 \%)\end{array}$ & $29(14.64 \%)$ \\
\hline $\begin{array}{l}\text { A woman can enter } \\
\text { kitchen/cook food } \\
\text { during menstruation }\end{array}$ & $07(3.53 \%)$ & $44(22.22 \%)$ & $50(25.0 \%)$ & $\begin{array}{l}83 \\
(41.91 \%)\end{array}$ & $16(8.08 \%)$ \\
\hline $\begin{array}{l}\text { A woman can take } \\
\text { bath during } \\
\text { menstruation }\end{array}$ & $11(5.55 \%)$ & $19(9.59 \%)$ & $32(16.0 \%)$ & $\begin{array}{l}108 \\
(54.54 \%)\end{array}$ & $30(15.15 \%)$ \\
\hline $\begin{array}{l}\text { A woman can wash } \\
\text { hair during } \\
\text { menstruation sleep }\end{array}$ & $13(6.56 \%)$ & $18(9.09 \%)$ & $19(9.5 \%)$ & $\begin{array}{l}121 \\
(61.1 \%)\end{array}$ & $29(14.64 \%)$ \\
\hline $\begin{array}{l}\text { A woman can sam bamed as } \\
\text { on same buring } \\
\text { others } \\
\text { menstruation }\end{array}$ & $11(5.55 \%)$ & $27(13.63 \%)$ & $33(16.5 \%)$ & $\begin{array}{l}100 \\
(50.50 \%)\end{array}$ & $29(14.64 \%)$ \\
\hline $\begin{array}{l}\text { A woman can touch } \\
\text { pickle during } \\
\text { menstruation }\end{array}$ & $08(4.04 \%)$ & $37(18.68 \%)$ & $17(8.68 \%)$ & $\begin{array}{l}103 \\
(52.02 \%)\end{array}$ & $35(17.5 \%)$ \\
\hline $\begin{array}{l}\text { A woman need not } \\
\text { avoid any foods } \\
\text { during menstruation }\end{array}$ & $23(11.5 \%)$ & $62(31.31 \%)$ & $20(10.1 \%)$ & $\begin{array}{l}82 \\
(41.41 \%)\end{array}$ & $13(6.5 \%)$ \\
\hline $\begin{array}{l}\text { A woman can have } \\
\text { sexual intercourse } \\
\text { during menstruation }\end{array}$ & $10(5.05 \%)$ & $97(48.98 \%)$ & $29(14.64 \%)$ & $\begin{array}{l}45 \\
(22.72 \%)\end{array}$ & $19(9.5 \%)$ \\
\hline
\end{tabular}

Table 5: Table demonstrating distribution of Practice regarding menstruation

\begin{tabular}{|l|r|l|l|l|l|}
\hline VARIALBE & Subgroups & $\begin{array}{l}\text { NUMBER } \\
\%\end{array}$ & $\begin{array}{l}\text { Good } \\
\text { practice } \\
\text { score }\end{array}$ & $\begin{array}{l}\% \\
\text { Good } \\
\text { practice }\end{array}$ \\
\hline $\begin{array}{l}\text { Soakage } \\
\begin{array}{l}\text { Material } \\
\text { Sentry }\end{array}\end{array}$ & II. Napkin $\quad$ Cloth & $\begin{array}{l}159(79.5) \\
41(20.5)\end{array}$ & 159 & 79.5 \\
\hline
\end{tabular}


Himalayan J. Soc. Sci. \& Humanities ISSN: 0975-9891

Vol. 16, (2021) 35-48

DOI: https://doi.org/10.51220/hjssh.v16i1.3

\begin{tabular}{|c|c|c|c|c|c|}
\hline $\begin{array}{ll}\text { Storage } & \text { of } \\
\text { soakage } & \\
\text { material } & \end{array}$ & $\begin{array}{l}\text { I. } \\
\text { II. } \\
\text { III. }\end{array}$ & $\begin{array}{l}\text { Unused cloths and pads clean (wrapped in } \\
\text { tissue or plastic bag) for further use. } \\
\text { Pads safely stored in the storage area. } \\
\text { Stored with other household material }\end{array}$ & $\begin{array}{l}133(66.5) \\
41(21) \\
26(13)\end{array}$ & 133 & 66.5 \\
\hline $\begin{array}{l}\text { Frequency } \\
\text { of changing } \\
\text { soakage } \\
\text { material }\end{array}$ & $\begin{array}{l}3-4 \mathrm{hrs} . \\
\text { After } 6 \mathrm{~h} \\
12 \mathrm{hrs} .\end{array}$ & & \begin{tabular}{|l|}
$103(51.5)$ \\
$51(26)$ \\
$46(23)$
\end{tabular} & 103 & 51.5 \\
\hline $\begin{array}{l}\text { Disposal of } \\
\text { soakage } \\
\text { material }\end{array}$ & $\begin{array}{l}\text { I. } \\
\text { II. }\end{array}$ & $\begin{array}{l}\text { Wrap a pad in a paper to make a clean package } \\
\text { in the bin so it can be burned later. } \\
\text { Disposed in an open dustbin }\end{array}$ & $\begin{array}{l}125(62.5) \\
75(37.5)\end{array}$ & 125 & 62.5 \\
\hline $\begin{array}{l}\text { Bathing } \\
\text { during } \\
\text { menstruation }\end{array}$ & $\begin{array}{l}\text { I. } \\
\text { II. } \\
\text { III. }\end{array}$ & $\begin{array}{l}\text { YES } \\
\text { OCCASIONALLY }\end{array}$ & $\begin{array}{l}131(65.5) \\
09(4.5) \\
60(30)\end{array}$ & 131 & 65.5 \\
\hline $\begin{array}{l}\text { Washing of } \\
\text { genitals after } \\
\text { micturition }\end{array}$ & $\begin{array}{l}\text { I. } \\
\text { II. } \\
\text { III. }\end{array}$ & $\begin{array}{l}\text { YES } \\
\text { OCCASIONALLY }\end{array}$ & $\begin{array}{l}145(72.5) \\
35(17.5) \\
20(10)\end{array}$ & 145 & 72.5 \\
\hline tal nerce & $\operatorname{scon}$ & practice & & & $66.33 \%$ \\
\hline
\end{tabular}

\section{Interrelationship between socio-demographic status and KAP}

Interrelationship between various socio-demographic variables with positive knowledge, attitude and practices involving menstruation is shown in Table 6 ( $\mathrm{P}$ values using One-way ANOVA)

Table 6:

\begin{tabular}{|r|l|l|l|}
\hline Socio-demographic variable & Knowledge & Attitude & Practice \\
\hline I. Age distribution: & & & \\
a. Less than 16 years & 0.43 & 0.401 & 0.5 \\
b. More than 16 years & 0.06 & 0.09 & 0.12 \\
\hline II. Level of education: & & & \\
a. Below high school & 0.07 & 0.08 & 0.56 \\
b. High school & 0.034 & 0.05 & 0.024 \\
c. Intermediate & 0.001 & 0.03 & 0.02 \\
a. $\geq 6391$ & 0.001 & & \\
b. 3196-6390 & 0.04 & 0.045 & 0.003 \\
c. 1917-3195 & 0.032 & 0.32 & 0.02 \\
d. 959-1916 & 0.23 & 0.56 & 0.03 \\
e. $\leq 958$ & 0.21 & 0.54 & 0.023 \\
\hline
\end{tabular}


Himalayan J. Soc. Sci. \& Humanities ISSN: 0975-9891

Vol. 16, (2021) 35-48

DOI: https://doi.org/10.51220/hjssh.v16i1.3

\begin{tabular}{|c|c|c|c|}
\hline $\begin{array}{l}\text { IV. Education status: } \\
\begin{aligned} \text { a. } & \text { Illiterate } \\
\text { b. } & \text { Primary level } \\
\text { c. } & \text { Middle school } \\
\text { d. } & \text { Secondary school }\end{aligned}\end{array}$ & $\begin{array}{l}1.2 \\
0.98 \\
0.82 \\
0.05\end{array}$ & $\begin{array}{l}1.9 \\
0.87 \\
0.65 \\
0.04\end{array}$ & $\begin{array}{l}2.1 \\
0.76 \\
0.04 \\
0.02 \\
\end{array}$ \\
\hline $\begin{array}{l}\text { V. Type of family: } \\
\text { a. Joint } \\
\text { b. Nuclear }\end{array}$ & $\begin{array}{l}0.4 \\
0.3\end{array}$ & $\begin{array}{l}0.23 \\
0.04\end{array}$ & $\begin{array}{l}0.03 \\
0.02\end{array}$ \\
\hline $\begin{aligned} \text { VI. Religion: } \\
\begin{aligned} & \text { a. } \text { Hindu } \\
& \text { b. } \text { Muslim } \\
& \text { c. } \text { Others } \\
&\end{aligned}\end{aligned}$ & $\begin{array}{l}0.02 \\
0.03 \\
0.01 \\
\end{array}$ & $\begin{array}{l}0.001 \\
0.003 \\
0.04\end{array}$ & $\begin{array}{l}0.71 \\
0.56 \\
0.34\end{array}$ \\
\hline $\begin{aligned} & \text { VII. Occupation of father: } \\
& \text { a. } \text { Private job } \\
& \text { b. } \text { Government job } \\
& \text { c. } \text { Self-employed } \\
&\end{aligned}$ & $\begin{array}{l}0.04 \\
0.02 \\
0.05 \\
\end{array}$ & $\begin{array}{l}0.04 \\
0.003 \\
0.002\end{array}$ & $\begin{array}{l}0.05 \\
0.003 \\
0.001 \\
\end{array}$ \\
\hline \begin{tabular}{ll} 
VIII. & \multicolumn{2}{l}{ Occupation of mother: } \\
a. & Private job \\
b. & Government job \\
c. & Self-employed \\
d. & Home-maker \\
\end{tabular} & $\begin{array}{l}0.56 \\
0.43 \\
0.24 \\
0.04\end{array}$ & $\begin{array}{l}0.12 \\
0.85 \\
0.34 \\
0.02\end{array}$ & $\begin{array}{l}0.03 \\
0.37 \\
0.23 \\
0.04\end{array}$ \\
\hline $\begin{aligned} & \text { IX. Primary source of informati } \\
& \text { a. } \text { Mother } \\
& \text { b. } \text { Sister } \\
& \text { c. } \text { Relative } \\
& \text { d. } \text { Neighbor } \\
& \text { e. } \text { Teacher }\end{aligned}$ & $\begin{array}{l}0.001 \\
0.02 \\
0.001 \\
1.6 \\
0.004\end{array}$ & $\begin{array}{l}0.02 \\
0.03 \\
1.3 \\
1.56 \\
0.003\end{array}$ & $\begin{array}{l}0.01 \\
0.002 \\
0.34 \\
0.51 \\
0.002\end{array}$ \\
\hline
\end{tabular}

Interrelationship between various socio-demographic variables with positive knowledge, attitude and practices involving menstruation: The overall good knowledge was found in $55.83 \%$ girls, While identifying the interrelation with age a significant association was found with age $(\mathrm{p}=0.06)$ and knowledge, also attitude and practice during menstruation were not associated with age of participant, student's education level also associated with their knowledge, attitude and practice, students in intermediate class have significant knowledge $(\mathrm{p}=0.001)$, attitude $(\mathrm{p}=0.003)$ and practice $(\mathrm{p}=0.02)$ towards menstruation. Socio-economic status of family was also associated with student's knowledge $(p=0.001)$, attitude $(p=0.045)$ and practice $(p=0.003)$. Education status of mother also affects the knowledge $(\mathrm{p}=0.05)$, attitude $(\mathrm{p}=0.04)$ and practice $(p=0.02)$ of girls during menstruation. Type of student's family does not affect the knowledge of girls during menstruation although it has significantly affected the attitude (nuclear family $\mathrm{p}=0.04$ ) and practice (nuclear family $\mathrm{p}=0.02$ ) of girls during menstruation. As per the study the 
Himalayan J. Soc. Sci. \& Humanities ISSN: 0975-9891

Vol. 16, (2021) 35-48

DOI: https://doi.org/10.51220/hjssh.v16i1.3

religion of student has not found significantly associated with attitude and practice toward menstruation, but the knowledge of girls were significantly associated with all the studied religions (Hindu, Muslim, others) in this study. Occupation of father was positively associated with knowledge, attitude and practice, while mothers who were housewives showed positive association with participant's knowledge attitude and practice. Girls who attained information from neighbours were not significant in their knowledge, attitude and practice, while remaining sources were significantly associated with their knowledge, attitude and practice.

\section{Discussion}

Menstruation leads to hormonal changes in the body, which converts a girl child into a woman who attained sexual maturity with physical and psychological changes (Rembeck et al 2006). It has been found in various studies that girls with proper knowledge about menstruation showed positive attitude towards menstruation also positive practice and hygiene, which is further responsible for better reproductive health of females, as unhygienic behaviour during menstruation leads to different infections, contributing to infertility later in their life (Dhingra et al 2009). In this study, the smallest girl was 12 year old and eldest girls were 18 year old. This study depicts that majority of students were more than 16 year in age i.e.71.5\%, these results are similar to other studies where most of participants were in their late menarche, reported in India (Jain et al 2017, Deshpande et al 2018). This study reported 88\% were Hindu; this result was also almost similar to the study reported in South India where 90.2\% participants were Hindu.

Educational level of mother determines the overall attitude of family regarding self-hygiene specifically adolescent girls; also mothers were the $1^{\text {st }}$ person who told girls about menstruation in this study and only $29.5 \%$ mothers were illiterate, so the mothers being $1^{\text {st }}$ resource person to the girls they must have educated the girls efficiently regarding basics of menstrual practices. Which indicates a positive attitude among girl students, the present study reported result were almost similar to previously reported studies in other parts of India (Geetha et.al. 2013, Omidvar, Begum 2011 ). Teacher's role for educating girls about menstruation was decreased to $26.76 \%$, which was not similar to studies reported in other countries where Teachers were $1^{\text {st }}$ resource person for educating girls about menstruation (Evans Paul 2016, Dhingra, Kumar 2009). 
Himalayan J. Soc. Sci. \& Humanities ISSN: 0975-9891

Vol. 16, (2021) 35-48

DOI: https://doi.org/10.51220/hjssh.v16i1.3

Different reported studies say that type of family also affects status of hygiene in the family; most of the girl students (66\%) were from joint family in this study, whereas different studies reported nuclear family set ups in India(Omidvar S, Begum 2011). This study also examined the socio-economic status of family which was found lower middle class for $34 \%$ students in this study. Previous studies reported in India showed lesser awareness about menstruation before achieving menarche, whereas $74 \%$ girl students in this study were aware about it before achieving menarche (Shanbhag et al 2012, Goel et al 2018). This awareness among girls indicates better knowledge related to menstruation practice.

In India, as a general practice, mothers are the one who first interact with girls about menarche and provide them sanitary material to handle their menstruation, this study also reported, mothers as primary source of information $(61.1 \%)$ about menstruation, similar to various other reported studies (Shanbhag et a, 2012, Goel et al 2018). Almost half (48.48\%) of Girl student's first reaction toward menstruation was usual in this study; probably because girls were already familiar with this process before reaching menarche. Only 25\% girls know about correct source of bleeding in spite of this $73.5 \%$ girls know this is a physiological process. $75 \%$ Girls have correct knowledge about normal interval of menstrual cycle, along with correct knowledge regarding infection occurrence due to poor menstrual hygiene, $70.5 \%$ girls answered correct about this question. Our study showed student's positive attitude during menstrual cycle in terms of entering kitchen, taking bath, hair wash and touching pickles, also they have a positive attitude to sleep with others during menses these findings were different from other reported studies in India (Deo, Ghattargi 2005 ). Students have negative attitude towards entering temples and sexual intercourse during menstruation. The study did not found any strict restrictions towards menstruation. These finding suggests the modifications in cultural beliefs regarding menstruation, which reflects better developed knowledge among girl students. Maintenance of hygienic practices during menstrual period save females from various reproductive infections, sexually transmitted diseases and urinary tract infections, which are responsible for life-threatening condition such as cervical cancers.

Overall good practice score for girls in our study was $66.33 \%$, which is far better than a study reported in Gujjar girls where only 3.1\% girls have good menstrual practice (Lawan et al 2010).Although these results were lower than previously reported studies in Ethiopia and North 
Himalayan J. Soc. Sci. \& Humanities ISSN: 0975-9891

Vol. 16, (2021) 35-48

DOI: https://doi.org/10.51220/hjssh.v16i1.3

western Nigeria, where (90.9\%) and (88.7\%) participants have good menstrual practice (Patavegar et al 2014). This study reported $79.5 \%$ girls used sanitary napkin whereas $20.5 \%$ girls were using cotton cloth for dealing their menstruation. This result was almost similar to a reported study (UNICEF. 2008), where $85.92 \%$ girls were using sanitary pads, it was better in comparison to a reported study in Delhi where only $60 \%$ girls were using sanitary napkins (Omidvar S, Begum 2011). Middle class socio-economic status of parents could be reason for the easy accessibility and affordability of disposable pads during menstruation. Girls have showed a satisfactory menstrual practice $(66.5 \%)$ regarding the storage of sanitary material both (disposable sanitary pads and cotton cloths) as per UNICEF guidelines (Pokhrel et a 2014). The frequency of changing sanitary napkins was also almost satisfactory as $51.5 \%$ girls were changing pads $3-4$ times in a day, which was a better practice than other reported studies(Dasgupta, Sarkar 2008).

The Girls in our study were not using proper practice regarding disposal of soakage material as only $61.5 \%$ girls were disposing pads in a proper package in the dustbin, later on which can be burned, this practice was similar to other reported studies(Sudeshna, Aparajita 2012 ). Other encountered practices were reported satisfactory, where $65.5 \%$ girls were allowed to bath during menstruation and $72.5 \%$ girls were washing their genitals after micturition. These self-hygiene practices are essential to maintain healthy urinary and reproductive conditions and also to protect themselves from various infections; which may be life threatening .

Our study also have limitations as this was carried out with a small data, the study was performed in only one government school level at urban area, which do not represent the complete perspective of whole state. A large data study (including rural, urban and town area of the state) could be conducted for better understanding of menstrual practice among adolescent girls.

\section{References:}

Aryeetey R, Ashinyo A, and Adjuik M, (2011) "Age of Menarche among basic level school girls in Madina, Accra," African Journal of Reproductive Health 15:3, 113-21.

Barathalakshmi J, Govindarajan PK, Ethirajan N, Felix AJ (2014). Knowledge and practice of menstrual hygiene among school going adolescent girls. Natl J Res Commun Med; $3: 138-42$.

Chumlea W. C. C, Schubert C. M., Roche A. F. et al., (2003) "Age at menarche and racial comparisons in US girls," Pediatrics, 111:1, 110-113. 
Himalayan J. Soc. Sci. \& Humanities ISSN: 0975-9891

Vol. 16, (2021) 35-48

DOI: https://doi.org/10.51220/hjssh.v16i1.3

Dambhare DG, Wagh SV. and Dudhe J. Y (2012) "Age at menarche and menstrual cycle pattern among school adolescent girls in Central India," Global Journal of Health Science, 4:1, 10511.

Dasgupta A and Sarkar M, 2008“Menstrual hygiene: how hygienic is the adolescent girl?" Indian Journal of Community Medicine , 33:2,77-80.

Dasgupta A, Sarkar M. 2008 Menstrual hygiene: How hygienic is the adolescent girl? Indian J Commun Med 33:77.

Deo DS, Ghattargi CH. (2005)Perceptions and practices regarding menstruation. A comparative study in urban and rural adolescent girls. Indian J Comm Med;30:33-4.

Deshpande TN, Patil SS, Gharai SB, Patil SR, Durgawale PM (2018). Menstrual hygiene among adolescent girls - A study from urban slum area. J Family Med Prim Care, 7:1439-45.

Deshpande TN, Patil SS, Gharai SB, Patil SR, Durgawale PM. (2018) Menstrual hygiene among adolescent girls - A study from urban slum area. J Family Med Prim Care; 7:1439-45.

Dhingra R, Kumar A, and Kour M (2009), "Knowledge and practices related to menstruation among Tribal (Gujjar) adolescent girls," Studies on Ethno-Medicine 3: 1, 43-48.

Dhingra R, Kumar A. (2009) Knowledge and practices related to menstruation among tribal (Gujjar) adolescent girls. Etho-Med. 3(1):43-8.

Evans Paul Kwame Ameade and Helene Akpene Garti.(2016) Relationship between female university students' knowledge on menstruation and their menstrual hygiene practices: a study in tamale, Ghana. Hindawi Publishing Corporation Advances in Preventive Medicine Volume, 10 pages.

Geetha P, Chenchuprasad C, Sathyavathi RB, Bharathi T, Reddy SK, et al. (2016) Effect of socioeconomic conditions and lifestyles on menstrual characteristics among rural women. $\mathrm{J}$ Women's Health Care, 5:298.

Goel P, Kumar R, Meena GS, Garg S. (2018) Association of sociodemographic characteristics with KAP regarding menstrual hygiene among women in an urban area in Delhi. Trop J Obstet Gynaecol, 35:158-64.

Goel P, Kumar R, Meena GS, Garg S. (2018) Association of sociodemographic characteristics with KAP regarding menstrual hygiene among women in an urban area in Delhi. Trop J Obstet Gynaecol, 35:158-64

Gultie T, Hailu D, and Workineh Y, (2014) "Age of menarche and knowledge about menstrual hygiene management among adolescent school girls in Amhara province, Ethiopia: implication to health care workers \& school teachers," PLoS ONE, 9: 9.

Gultie T, Hailu D, Workineh Y (2014). Age of menarche and knowledge about menstrual hygiene management among adolescent school girls in Amhara province, Ethiopia: Implication to health care workers \& school teachers. PLoS One ,9:e108644.

Jain R, Anand P, Dhyani A, Bansai D. (2017) Knowledge and awareness regarding menstruation and HIV/AIDS among school going adolescent girls. J Family Med Prim Care, 6:47-51

K. A. Tunau, A. N. Adamu, M. A. Hassan, Y. Ahmed, and B. A. Ekele, (2012) "Age at menarche among school girls in Sokoto,Northern Nigeria," Annals of African Medicine , 11:2,. 103-07.

Kumbhar SK, Reddy M, Sujana B, Roja RK. and Balkrishna C, (2011) "Prevalence of dysmenorrhea among adolescent girls (14-19 yrs.) of Kadapa district and its impact on quality of life: a cross sectional study," National Journal of Community Medicine, 2:2, 26568. 
Lawan U, Nafisa W, Musa A. (2010) Menstruation and menstrual hygiene amongst adolescent school girls in Kano Northwestern Nigeria. Afr J Reprod Health. 14(3):201-7.

Omidvar S, Begum K. (2011)Menstrual pattern among unmarried women from south India. J Nat Sci Biology Med, 2:174.

Patavegar BN, Kapilashrami MC, Rasheed N, Pathak R. (2014) Menstrual hygiene among adolescent school girls: An in-depth cross-sectional study in an urban community. Int $\mathbf{J}$ Health Sci Res 4:15-21.

Pokhrel S, Mahantashetti N, Angolkar M, and Devkota N, (2014) "Impact of health education on knowledge, attitude and practice regarding menstrual hygiene among pre-university female students of a college located in urban area of Belgaum," Journal of Nursing and Health Science, vol. 3, no. 4, pp. 38-44,.

Poureslami M and Osati-Ashtiani F, (2002) "Assessing knowledge, attitudes and behaviour of adolescent girls in sub-urban districts of Tehran about dysmenorrhea and menstrual hygiene," Journal of International Women's Studies, 3: 2, 51-61.

Reid G, Bruce AW (2003). Urogenital infections in women: can probiotics help? Postgrad Med J. 79:428-32.

Rembeck GI, Moller M, and Gunnarsson R (2006) "Attitudes and feelings towards menstruation and womanhood in girls at menarche," Acta Paediatrica, 95: 6, 707-14.

Shanbhag D, Shilpa R, D’Souza N, Josephine P, Singh J, and Goud BR (2012), "Perceptions regarding menstruation and practices during menstrual cycles among high school going adolescent girls in resource limited settings around Bangalore city, Karnataka, India," International Journal of Collaborative Research on Internal Medicine and Public Health 2012, 4: 7,1353-62.

Sharma S, Mehra D, Kohli C, Singh MM. (2017) Menstrual hygiene practices among adolescent girls in a resettlement colony of Delhi: A cross-sectional study. Int J Reprod Contracept Obstet Gynecol, 6:1945-51.

Sommer M, Sahin M. (2013) Overcoming the taboo: Advancing the global agenda for menstrual hygiene management for school-girls. Am J Public Health; 103:1556-9.

Sudeshna R, Aparajita D. 2012 Determinants of menstrual hygiene among adolescent girls: A multivariate analysis. Natl J Community Med, 3:294-301.

Thomas F, Renaud F, Benefice E, DeMee"us T., and Guegan J.-F (2001) "International variability of ages at menarche and menopause: patterns and main determinants," Human Biology, 73:2, 271-290.

UNESCO, (2014) "Good policy and practice in health education: puberty education and menstrual hygiene management- Booklet 9," UNESCO, , http://unesdoc.unesco.org/images/ 0022/002267/226792e.pdf.

UNICEF. 2008 Sharing Simple Facts: Useful Information about Menstrual Health and Hygiene. New Delhi.

Yasmin S, Manna N, Mallik S, Ahmed A, Paria B. (2013) Menstrual hygiene among adolescent school students: An in-depth cross-sectional study in an urban community of West Bengal, India. IOSR J Dent Med Sci; 5:22-6. 